2012s-18

\title{
Ambiguïté, identification partielle et politique environnementale
}

Alfred Galichon, Marc Henry

\begin{tabular}{c}
\hline Série Scientifique \\
Scientific Series
\end{tabular}

\author{
Montréal \\ Juin 2012
}

(C) 2012 Alfred Galichon, Marc Henry. Tous droits réservés. All rights reserved. Reproduction partielle permise avec citation du document source, incluant la notice (C).

Short sections may be quoted without explicit permission, if full credit, including (C) notice, is given to the source.
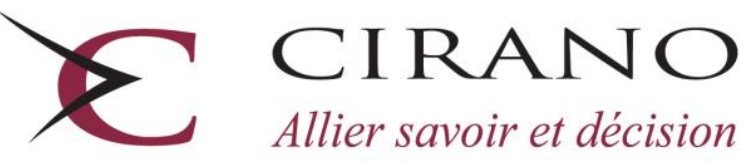

Allier savoir et décision

Centre interuniversitaire de recherche en analyse des organisations 


\section{CIRANO}

Le CIRANO est un organisme sans but lucratif constitué en vertu de la Loi des compagnies du Québec. Le financement de son infrastructure et de ses activités de recherche provient des cotisations de ses organisations-membres, d'une subvention d'infrastructure du Ministère du Développement économique et régional et de la Recherche, de même que des subventions et mandats obtenus par ses équipes de recherche.

CIRANO is a private non-profit organization incorporated under the Québec Companies Act. Its infrastructure and research activities are funded through fees paid by member organizations, an infrastructure grant from the Ministère du Développement économique et régional et de la Recherche, and grants and research mandates obtained by its research teams.

\section{Les partenaires du CIRANO}

\section{Partenaire majeur}

Ministère du Développement économique, de l'Innovation et de l'Exportation

\section{Partenaires corporatifs}

Autorité des marchés financiers

Banque de développement du Canada

Banque du Canada

Banque Laurentienne du Canada

Banque Nationale du Canada

Banque Royale du Canada

Banque Scotia

Bell Canada

BMO Groupe financier

Caisse de dépôt et placement du Québec

Fédération des caisses Desjardins du Québec

Financière Sun Life, Québec

Gaz Métro

Hydro-Québec

Industrie Canada

Investissements PSP

Ministère des Finances du Québec

Power Corporation du Canada

Rio Tinto Alcan

State Street Global Advisors

Transat A.T.

Ville de Montréal

\section{Partenaires universitaires}

École Polytechnique de Montréal

HEC Montréal

McGill University

Université Concordia

Université de Montréal

Université de Sherbrooke

Université du Québec

Université du Québec à Montréal

Université Laval

Le CIRANO collabore avec de nombreux centres et chaires de recherche universitaires dont on peut consulter la liste sur son site web.

Les cahiers de la série scientifique (CS) visent à rendre accessibles des résultats de recherche effectuée au CIRANO afin de susciter échanges et commentaires. Ces cahiers sont écrits dans le style des publications scientifiques. Les idées et les opinions émises sont sous l'unique responsabilité des auteurs et ne représentent pas nécessairement les positions du CIRANO ou de ses partenaires.

This paper presents research carried out at CIRANO and aims at encouraging discussion and comment. The observations and viewpoints expressed are the sole responsibility of the authors. They do not necessarily represent positions of CIRANO or its partners. 


\title{
Ambiguïté, identification partielle et politique environnementale
}

\author{
Alfred Galichon", Marc Henry"
}

\section{Résumé / Abstract}

Cet article illustre le lien entre identification partielle dans les modèles économétriques et critère de décision de Jaffray dans l'incertain non probabilisé à travers le problème du choix de niveau optimal d'émissions toxiques dans un lac partagé entre deux communes.

Mots clés/Keywords: : Décision en environnement incertain, identification partielle, fonction de croyance, pollution, réchauffement climatique, taux d'escompte, valorisation des biens environnementaux.

Codes JEL : C18, D81, Q51

\footnotetext{
*École Polytechnique, Paris - Département de Sciences économiques.

${ }^{\dagger}$ CIRANO et Université de Montréal. Adresse de correspondance: Département de sciences économiques, Université de Montréal, C.P. 6128, succursale Centre-ville, Montréal, Québec, H3C 3J7, Canada, marc.henry@umontreal.ca.
} 


\section{INTRODUCTION}

Dans son discours présidentiel de 1970 à Cambridge, Jacques Drèze [4] identifie quatre étapes dans la résolution d'un problème de politique publique à la lumière du théorème de Savage [16]: 1. La détermination des actes à la disposition du décideur, 2. le choix d'une fonction d'utilité sur les conséquences, 3. l'estimation d'une fonction de probabilité sur les états du monde et 4 . la détermination de l'acte qui maximise l'espérence d'utilité. Il met l'accent tout particulièrement sur le point 3. de ce programme et c'est ce point qui pose des difficultés particulière en analyse de politiques environnementales. L'analyse coûts-bénéfices environnementale fait intervenir de nombreux éléments dont notre connaissance est incomplète. La politique de contrôle des émissions de gaz à effet de serre, pour citer un exemple amplement documenté, repose sur l'évaluation des dommages qu'ils causeront à moyen, long et très long terme. La connaissance scientifique existante, résumée dans les rapports successifs du groupe d'experts intergouvernementaux sur l'évolution du climat, ne permet à l'évidence pas de résumer l'incertitude sur les dommages sous la forme d'une distribution de risque. Nous sommes bien là dans une situation d'incertitude non probabilisée, ou ambiguïté. Outre la connaissance imparfaite de l'effet de la concentration atmosphérique de gaz à effet de serre sur le climat et du climat sur le capital naturel, l'ambiguïté affecte la politique environnementale à travers les quantités suivantes.

- Le taux de rendement social du capital physique. L'analyse de Weitzman [19] met bien en évidence l'incertitude qui entoure la valeur du taux d'escompte. Dans un modèle de croissance à la Solow-Ramsey et abstraction faite de préférence pure pour le present, le taux de rendement social du capital physique suit la formule de Ramsey $r=g \sigma$, où $g$ mesure le progrès technique et $\sigma$ l'élasticité de l'utilité marginale. La source d'ambiguïté sur $r$ est double. L'ambiguïté scientifique, d'une part, dûe à notre capacité limitée à prévoir le taux d'accroissement $g$ du capital physique à long terme et l'ambiguité que nous appellerons économique, d'autre part, dûe à notre connaissance incomplète des préférences et donc de la valeur de $\sigma$.

- La substituabilité entre capital physique et capital naturel. Les modèles de croissance à deux biens adoptés par Henry [9] et Guesnerie [8], sur la base des travaux de Malinvaud [13], mettent clairement en évidence le rôle crucial joué par la substituabilité entre capital naturel et capital artificiel. Là encore, deux sources d'ambiguïté se combinent. À l'ambiguïté scientifique relative à notre capacité à substituer le capital naturel par du capital artificiel (ou de manière équivalente, notre capacité à réparer les dégradations subies par le capital naturel) s'ajoute l'ambiguité économique relative à notre propension à nous satisfaire de tels substituts. 
- Le bien-être émanant du capital naturel. À l'ambiguïté scientifique évoquée plus haut relative à l'évaluation des dégradations du capital naturel s'ajoute encore l'ambiguïté économique relative à la valorisation des biens et des services environnementaux dégradés, dont il sera question plus en détail dans la prochaine section.

Comme le soulignent Henry et Henry [10], l'ambiguïté décrite ci-dessus rend l'analyse coûts-bénéfices par le critère d'espérence d'utilité classique inapplicable. Il existe une vaste littérature et de nombreux critères de décision en présence d'ambiguïté, dont une part, en commençant par Jaffray [12], met l'accent sur l'articulation entre le critère de décision et des structures particulières d'information objective ambigüe, en particulier des fonctions de croyance de Dempster [3]. Nous montrons ici que cette structure particulière d'information objective s'obtient lorsque l'information est le produit d'estimation statistique à l'aide de modèles partiellement identifiés. En cela nous rejoignons le désir formulé par Drèze [4] de marier économétrie et théorie de la décision.

Formellement, une source d'aléa probabilisé et un paramètre inconnu $\theta$ produisent des observations $Y$. Les données manquantes, censurées, erreurs de mesure, interactions, sources d'endogénéité et spécification incomplète du modèle empêchent d'identifier une valeur unique du paramètre inconnu $\theta$. Le modèle est alors appelé partiellement identifié et toutes les valeurs du paramètre $\theta$ dans un ensemble de paramètres $\Theta_{I}$, appelé ensemble identifié, sont observationnellement équivalentes. L'inférence statistique permet l'estimation de $\Theta_{I}$, qui, couplé à l'aléa probabilisé, produit l'information objective sur laquelle est basé le critère de décision. Cette dernière est ainsi sous la forme d'une fonction de croyance, proposée par Dempster [3] pour généraliser l'inférence Bayesienne. Un des premiers modèles formalisés de décision dans l'incertain non probabilisé est celui de Jaffray [12], qui propose un critère de décision en presence d'information objective sous cette forme. Il reformule le problème de décision dans l'incertain non probabilisé en un problème de choix entre correspondances (ou fonctions à valeurs multiples) en environnement risqué et propose un critère simple d'espérance d'utilité pour ce choix entre correspondences.

Nous illustrons cette relation entre identification partielle et le critère de décision de Jaffray [12] à travers un exemple stylisé de décision en politique environnementale. En section 1, nous illustrons chacune des sources d'ambiguïté, économique et scientifique, à travers des modèles partiellement identifiés simples et en section 2 , nous explicitons la fonction de croyance résultante et le critère de décision correspondant. 
TABLE 1. Fonctions de payement des communes

\begin{tabular}{l|cc|cc} 
& $Y_{2}=0$ & \multicolumn{2}{|c}{$Y_{2}=1$} \\
\hline$Y_{1}=0$ & 0 & 0 & 0 & $v(0)-\varepsilon_{2}$ \\
$Y_{1}=1$ & $v(0)-\varepsilon_{1}$ & 0 & $v(1)-\varepsilon_{1}$ & $v(1)-\varepsilon_{2}$
\end{tabular}

\section{AmbiguïtÉ ET IDENTIFICATION PARTIELlE DANS LES MODÈLES ÉCONOMÉTRIQUES}

Dans ce qui suit, nous prenons pour exemple un problème de décision environnementale stylisé où l'ambiguïté, à la fois économique et scientifique, relève de la valorisation d'un lac partagé entre deux communes, du coût de réduction d'émissions toxiques dans le lac et de l'effet des émissions sur la santé écologique de ce dernier. Les communes font chacune le choix d'utiliser le lac à des fins récréatives ou à des fins polluantes. Un régulateur doit décider du niveau maximum d'émissions polluantes autorisé dans le lac. Les éléments pris en compte sont le coût de réduction des émissions, l'effet des émissions sur la santé écologique du lac et la valorisation d'un lac écologiquement sain par les communes. Considérons ces éléments successivement.

\subsection{Valorisation du lac.}

La littérature concernant la valorisation des biens environnementaux est vaste et le but n'est pas d'en faire ici une revue exhaustive, mais de montrer que la structure économétrique des modèles utilisés conduit à une structure particulière de la connaissance. Nous montrons que l'identification partielle des modèles économétriques, que nous définirons et discuterons plus avant, conduit naturellement à une modélisation de l'incertitude sur la valeur des biens environnementaux non sous la forme d'une loi de probabilité, mais sous la forme d'une fonction de croyance à la Dempster [3], qui en est une généralisation.

Prenons tout d'abord le cas de la méthode des préférences révélées, qui se trouve être emblématique dans ce contexte. Outre des variables socio-économiques telles la population et le revenu moyen des communes, le régulateur observe le choix d'usage du lac, sous la forme d'une variable $Y_{i}=1$ si la commune $i$ fait un usage récréatif du lac, et $Y_{i}=0$ sinon. L'interaction entre les deux communes est modélisée sous la forme d'un jeu non coopératif en forme normale avec les fonctions de payement (qui dépendent implicitement des variables socio-économiques, population, revenu etc...) données dans la table 1. L'utilité de la commune $i=1,2$, est normalisée à 0 si la commune exclut l'usage récréatif du lac $\left(Y_{i}=0\right)$. Si la commune choisit l'usage récréatif $\left(Y_{i}=1\right)$, son utilité est égale à la différence de deux termes: d'une part, une fonction des variables explicatives $v(1)$ lorsque la commune voisine choisit l'usage récréatif et $v(0)$ sinon et une composante aléatoire $\varepsilon_{i}$ d'autre part. On suppose que les communes choisissent l'option qui maximise leurs utilités respectives à l'équilibre 
de Nash en stratégies pures. Notons, cependant, que le choix de modélisation de l'interaction entre les communes, s'il est simple et en accord avec la littérature, n'est nullement nécessaire à la thèse défendue ici. Des résultats qualitativement équivalents seraient obtenus avec d'autres choix de concepts d'équilibre (Nash en stratégies mixtes, stratégies corrélées, solutions coopératives etc...). Le modèle peut donc se réécrire sous la forme

$$
Y_{i}=1\left\{v\left(Y_{3-i}\right) \geq \varepsilon_{i}\right\}, \quad i=1,2,
$$

où $1\{$.$\} dénote la fonction indicatrice et la loi marginale de \varepsilon_{i}$ peut être supposée uniforme sur $[0,1]$ sans perte de généralité. L'ensemble des équilibres de Nash en stratégies pures (la correspondance d'équilibre) est fonction des composantes aléatoires $\left(\varepsilon_{1}, \varepsilon_{2}\right)$ et peut se représenter sur $[0,1]^{2}$ comme en figure 1. On suppose que $v(1)>v(0)$, autrement dit que l'usage récréatif procure une utilité plus grande si le lac est propre. Lorsque le choc négatif d'utilité est suffisemment faible, soit $\varepsilon_{i} \leq v(0)$, la meilleure réponse de la commune $i$ est toujours l'usage récréatif. Inversement, si $\varepsilon_{i}>v(1)$, la meilleure réponse de la commune $i$ est toujours l'usage polluant. Une caractéristique saillante de la correspondance d'équilibre représentée en figure 1 est la multiplicité d'équilibres lorsque les chocs d'utilité sont dans la région $[v(0), v(1)]^{2}$. Si la commune voisine choisit l'usage récréatif, la meilleure réponse est aussi l'usage récréatif. Inversement, si la commune voisine choisit l'usage polluant, la meilleure réponse est aussi l'usage polluant.

FiguRE 1. Correspondance d'équilibre.

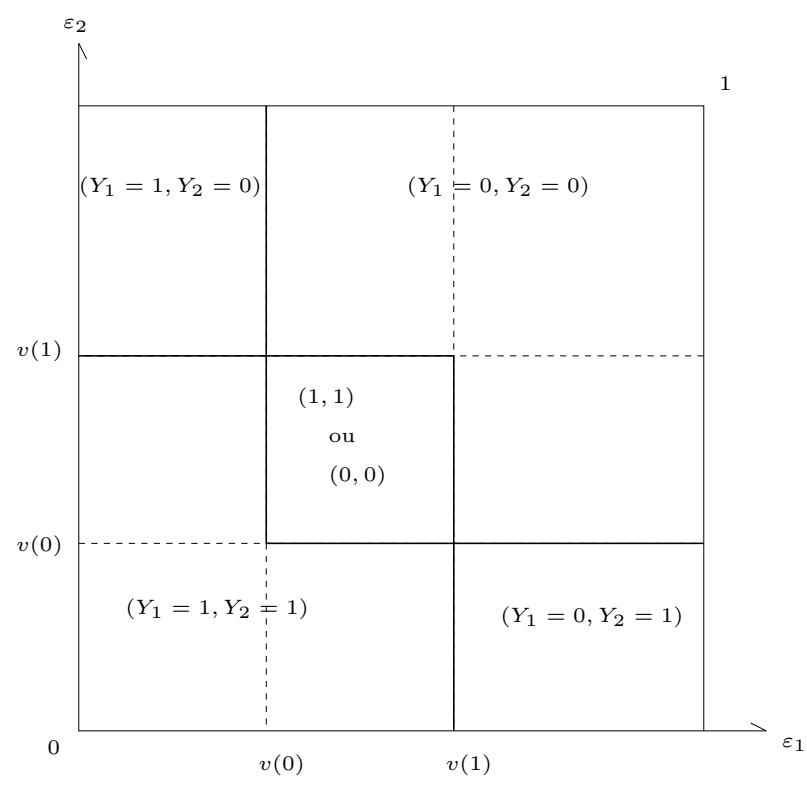


La multiplicité d'équilibres, très fréquente dans les modèles d'interaction, est une des sources d'ambiguïté sur la valorisation du lac par les communes, comme nous allons le montrer ci-dessous. Le modèle économétrique (1.1) est un modèle de choix discret. Traditionnellement, l'estimation des fonctions $v(0)$ et $v(1)$ s'effectuerait à l'aide de la vraisemblance d'un échantillon de paires de communes qui partagent un lac. Cette méthode est inapplicable ici pour deux raisons. D'une part, la loi de probabilité jointe de la paire $\left(\varepsilon_{1}, \varepsilon_{2}\right)$ est inconnue. Le modèle prédit par exemple une fréquence de situations où la commune 1 fait un usage récréatif du lac et la commune 2 en fait un usage polluant (observation $Y_{1}=1, Y_{2}=0$ ) égale à la probabilité $\operatorname{Pr}\left(\varepsilon_{1} \leq v(0), \varepsilon_{2}>v(1)\right.$ ), qui est inconnue, mais qui peut être bornée par

$$
0 \leq \operatorname{Pr}\left(\varepsilon_{1} \leq v(0), \varepsilon_{2}>v(1)\right) \leq 1-v(1)
$$

qui sont les bornes de Fréchet [5]. D'autre part, même dans le cas où la loi jointe des utilités inobservées $\left(\varepsilon_{1}, \varepsilon_{2}\right)$ est connue, la prédiction de la fréquence de situations où les deux communes choisissent l'usage récréatif, par exemple, nécessite des hypothèses supplémentaires sur le mécanisme de sélection d'équilibre. Dans l'ignorance, hypothèse réaliste, du mécanisme de sélection d'équilibre, les prédictions du modèle permettent seulement de borner la fréquence $p_{11}=\operatorname{Pr}\left(Y_{1}=Y_{2}=1\right)$ par

$$
\operatorname{Pr}\left(\varepsilon_{1} \leq v(1), \varepsilon_{2} \leq v(0)\right)+\operatorname{Pr}\left(\varepsilon_{1} \leq v(0), v(0)<\varepsilon_{2} \leq v(1)\right) \leq p_{11} \leq \operatorname{Pr}\left(\varepsilon_{1} \leq v(1), \varepsilon_{2} \leq v(1)\right) .
$$

En effet, il se peut que toutes les paires de communes telles que $\left(\varepsilon_{1}, \varepsilon_{2}\right) \in[v(0), v(1)]^{2}$ choisissent l'usage récréatif, ou bien aucune, ou bien une fonction inconnue de $\left(\varepsilon_{1}, \varepsilon_{2}\right)$. Dans les deux cas, méconnaissance de la loi jointe des composantes inobservables de l'utilité d'une part et équilibres multiples d'autre part, le modèle peut être complété pour en rétablir la cohérence, selon la terminologie de Gouriéroux, Laffont et Monfort [7]. D'une part, l'analyste peut faire l'hypothèse d'une loi paramétrique pour les composantes inobservables $\left(\varepsilon_{1}, \varepsilon_{2}\right)$. D'autre part, l'analyste peut faire l'hypothèse d'un mécanisme de sélection d'équilibre. Ces deux hypothèses conjointes complètent le modèle et permettent l'estimation par maximum de vraisemblance, au prix d'hypothèses fortes et difficilement justifiables. L'autre branche de l'alternative, préconisée en particulier par Manski [14], consiste à éviter les hypothèses d'identification ad hoc et à proposer des bornes pour les fonctions d'intérêt $v(0)$ et $v(1)$. Dans cette optique, Galichon et Henry [6] proposent des méthodes de théorie du transport optimal (voir par exemple Villani [18]) permettant la construction de bornes pour les paramètres de modèles avec équilibres multiples qui en épuisent le contenu empirique. Ainsi toutes les valeurs des paramètres qui satisfont les bornes sont observationnellement équivalentes et aucune ne peut être rejetée sans information supplémentaire a priori. Galichon et Henry [6] montrent que la caractérisation de cet ensemble de valeurs compatibles peut se ramener à un problème classique 
d'appariement, pour la résolution duquel de nombreux algorithmes ont été développés et appliqués en économie et en recherche opérationnelle.

Dans le cas présent, les bornes obtenues par la méthode de transport optimal sont les suivantes.

$$
\begin{aligned}
\max \left(p_{10}, p_{0,1}\right) & \leq v(0) \leq p_{11}+\min \left(p_{10}, p_{01}\right) \\
p_{11}+\max \left(p_{10}, p_{0,1}\right) & \leq v(1) \leq 1-\max \left(p_{10}, p_{01}\right)
\end{aligned}
$$

où les $p_{i j}=\operatorname{Pr}\left(Y_{1}=i, Y_{2}=j\right.$ ) sont les fréquences réelles (qui seraient obtenues avec un échantillon de taille infinie). Les bornes ci-dessus caractérisent ce qui peut être déduit du modèle sur la valorisation du lac par les communes. Cette situation, où les valeurs de $v(0)$ et $v(1)$ ne peuvent être connues exactement, même avec un échantillon de taille infinie, est appelée identification partielle. Nous verrons plus loin que l'identification partielle du modèle économétrique produit une structure d'information appelée fonction de croyance de Dempster [3].

L'exposé ci-dessus s'est focalisé sur un exemple d'élicitation par la méthode des préférences révélées. Cependant, tout modèle économétrique d'élicitation de préférences pour la valorisation des biens et des services environnementaux est sujet à l'identification partielle. L'accent a été placé sur les équilibres multiples et la corrélation entre composantes inobservables de l'utilité, mais les données manquantes ou censurées ainsi que les erreurs de mesures, fréquentes en évaluation contingente, sont aussi des sources d'identification partielle de la valorisation des biens environnementaux (voir [6] pour plus de détails).

\subsection{Coût de réduction des émissions toxiques.}

Supposons que la politique du régulateur consiste à limiter les émissions polluantes au niveau $e$. Appelons $c(e)$ la fonction de coût de réduction des émissions jusqu'au niveau $e$. Un raisonnement similaire à celui que nous avons conduit dans la section 1.1 permet de montrer que les modèles économétriques utilisés pour estimer les coûts de réduction d'émissions (voir par exemple Schmalensee [17] ou Hourcade et al. [11] pour le cas des émissions de gaz à effet de serre) sont fréquemment partiellement identifiés du fait de données manquantes, d'erreurs de mesure et de composantes inobservables incomplètement spécifiées. Dans le cas particulier de nos deux communes, $c(e)$ est le manque à gagner fiscal dû à la réduction d'émissions. Ce manque à gagner peut être estimé à l'aide d'un modèle économétrique simple et sur la base d'observations passées de niveaux d'émissions et de niveaux d'impositions d'établissements polluants. Soit un échantillon de $n$ communes et $T_{i}, i=1, \ldots, n$, les recettes fiscales de la commune $i, E_{i}^{*}$ le niveau d'émissions, $X_{i}$ un vecteur de caractéristiques observables de la commune. Supposons que le modèle économétrique stipule la relation suivante:

$$
T_{i}=\alpha E_{i}^{*}+\beta X_{i}+u_{i}
$$


où $\alpha$ est un paramètre inconnu qui mesure le coût de réduction des émissions, $\beta$ est un vecteur de paramètres et $u_{i}$ un terme d'erreur, ou hétérogénéité inobservée, indépendente de $E_{i}^{*}$ et $X_{i}$. Les données sur les émissions effectives sont souvent mesurées avec erreur ou bien sont censurées, par exemple si l'on observe le maximum légal $E_{i}$ au lieu des émissions effectives $E_{i}^{*}$. Dans ces conditions, le paramètre d'intérêt $\alpha$ est partiellement identifié: un échantillon de taille infinie permettrait au mieux de déduire $\alpha \in[\underline{\alpha}, \bar{\alpha}]$. Le théorème 1 de [6] permet de déterminer $\underline{\alpha}$ et $\bar{\alpha}$. En conséquence, la connaissance du régulateur se réduit donc à une fourchette de coûts $[\underline{c}(e), \bar{c}(e)]$ de réduction des émissions.

\subsection{Effet des émissions sur la santé écologique du lac.}

Si l'ambiguïté relative aux préférences et aux coûts de réduction d'émissions est essentiellement économique et technique, l'ambiguité relative aux dégradations du capital naturel est exclusivement physique. Cependant, les méthodes empiriques d'élicitation d'information ne sont pas radicalement différentes des méthodes économétriques évoquées ci-dessus. L'articulation entre ambiguïté et identification partielle est aussi pertinente pour la modélisation de l'incertitude scientifique quant aux effets des émissions toxiques sur la santé écologique du lac. Considérons deux états possibles pour le lac: un état écologiquement sain qui permet son usage récréatif et un état eutophisé, qui ne le permet plus. L'analyse de l'incertitude scientifique relative à l'eutrophisation d'un lac proposé par Capenter, Ludwig et Brock [1] mène à une modélisation de l'incertitude qui se résume de la manière suivante. L'eutrophisation intervient à courte échéance avec une probability $\bar{F}(e)$ si le flux d'émissions $e$ est compris entre $\underline{e}$ et $\bar{e}$.

\section{Ambiguïté et déCision: AU delÀ de L’espérance D'Utilité}

Le problème de décision environnementale se résume de la manière suivante.

- Le régulateur cherche à déterminer le niveau optimal d'émissions polluantes dans le lac.

- Il détient une information ambigüe sur la fonction de coût de réduction d'émissions, qui est comprise entre $\underline{c}(e)$ et $\bar{c}(e)$.

- Si le lac reste écologiquement sain, l'utilité qu'en retirent les communes est comprise entre $\underline{v}$ et $\bar{v}$.

- L'eutrophisation du lac intervient pour un niveau d'émissions compris entre $\underline{e}$ et $\bar{e}$ avec une probabilité $\bar{F}(e)$.

L'incertitude qui résulte de l'estimation économétrique des préférences, des coûts de réduction et des caractéristiques écologiques du lac n'est pas modélisée sous la forme d'une loi de probabilité. Le critère d'espérance d'utilité n'est donc pas directement applicable. En revanche, la structure des 
modèles économétriques partiellement identifiés produit une modélisation de l'incertitude sous la forme d'une fonction de croyance à la Dempster [3] et le critère de décision en présence de fonctions de croyance dû à Jaffray [12] est directement applicable. Nous proposons ici une courte présentation de la notion de fonction de croyance et du critère de décision de Jaffray.

\subsection{Fonction de croyance.}

La fonction de croyance est proposée par Dempster [3] comme modélisation de l'incertitude non probabilisée. Sa structure mathématique est celle d'un ensemble aléatoire (voir Matheron [15]) ou encore d'une capacité de Choquet infiniment alternée (voir Choquet [2]). Comme modélisation de l'incertitude, elle s'interprète de manière simple. Dans un problème de décision classique, on modélise l'incertitude par un ensemble d'états du monde $\mathcal{X}$. L'un des états est réalisé, mais cet état est inconnu. Une situation de risque est une situation où la probabilité de chacun des états est connue. Une fonction de croyance est une collection d'évènements $A_{i} \subseteq \mathcal{X}, i \in I$, dont la probabilité est connue.

Ainsi, dans notre problème de décision environnementale, chaque état du monde est un triplet $x=$ $(v, c, e)$ qui s'interprète de la manière suivante: dans l'état $x$, la valorisation d'un lac écologiquement sain est exactement $v$, la fonction de coûts de réduction d'émissions est précisément la fonction $c: \tilde{e} \mapsto$ $c(\tilde{e})$ et l'eutrophisation du lac intervient lorsque le niveau des émissions toxiques atteint exactement $e$. Les actes à la disposition du régulateur sont les fonctions ẽ (niveau maximal d'émissions autorisées) qui sont des fonctions définies sur $\mathcal{X}$ à valeurs:

$$
\tilde{e}:(v, c, e) \mapsto \tilde{e}(v, c, e)=v 1\{\tilde{e}<e\}-c(\tilde{e}) .
$$

En effet, dans l'état $x=(v, c, e)$, la valoristion du lac est $v$ si le niveau maximal d'émissions autorisées ne dépasse pas $e$ et zero sinon, tandis que le coût de réduction des émissions est $c(\tilde{e})$. La fonction de croyance qui résulte de l'évaluation économétriques des préférences, des coûts et des caractéristiques écologiques du lac consiste en une collection d'évènements $A(e) \subseteq \mathcal{X}$ définis de la manière suivante.

- $A(e)$ est l'ensemble des états du monde tels que l'eutrophisation du lac intervient entre le niveau $\underline{e}$ et le niveau $\bar{e}$, la valorisation d'un lac sain est comprise entre $\underline{v}$ et $\bar{v}$ et la fonction de coûts est comprise entre la fonction $\underline{c}$ et la fonction $\bar{c}$.

La probabilité $\bar{F}(e)$ de réalisation de l'évènement est connue et les actes $\tilde{e}: x \mapsto \tilde{e}(x)$ à la disposition du régulateur sont caractérisés par leurs valeurs minimale et maximale si $A(e)$ est réalisé.

$$
\tilde{e}_{\mid A(e)} \in[\underline{v} 1\{\tilde{e}<\underline{e}\}-\bar{c}(\tilde{e}), \bar{v} 1\{\tilde{e}<\bar{e}\}-\underline{c}(\tilde{e})] .
$$


En effet, dans le pire des états du monde contenus dans $A(e)$, l'eutrophisation intervient pour un niveau d'émissions $\underline{e}$, la valorisation d'un lac sain est $\underline{v}$ et le coût de réduction des émissions $\bar{c}(\tilde{e})$. Inversement, dans le meilleur des états du monde contenus dans $A(e)$, l'eutrophisation intervient pour le niveau d'émissions supérieur $\bar{e}$, la valorisation d'un lac sain est $\bar{v}$ et le coût de réduction des émissions est le plus faible, soit $\underline{c}(\tilde{e})$.

Le choix du niveau maximal légal d'émissions est donc caractérisé par une correspondence (fonction à valeurs multiples) et il nous faut donc recourir à un critère de choix entre correspondences. L'information peut se résumer de la manière suivante: l'acte ẽ produit un intervalle de conséquences

$$
[\underline{\tilde{e}}, \bar{e}]=[\underline{v} 1\{\tilde{e}<\underline{e}\}-\bar{c}(\tilde{e}), \bar{v} 1\{\tilde{e}<\bar{e}\}-\underline{c}(\tilde{e})]
$$

avec un probabilité $\bar{F}(e)$ connue. Il s'agit donc d'un choix en environnement risqué, mais un choix entre correspondences, et non entre fonctions (qui appellerait naturellement le critère d'espérance d'utilité).

\subsection{Critère de décision.}

Le critère de décision de Jaffray [12] s'applique précisément à cette situation où l'incertitude est présentée sous forme d'évènements probabilisés et en absence totale d'information sur la vraisemblance relative des états du monde que ces évènements contiennent. Comme on l'a vu, cette situation est équivalente à une situation où l'on choisit entre correspondances en environnement risqué. Jaffray [12] utilise cette représentation équivalente et propose un critère simple d'espérence d'utilité $\mathbb{E} \gamma(\tilde{e})$, où la fonction d'utilité s'applique à la correspondance ẽ et non à une fonction. Ainsi, $\gamma$ dépend des valeurs minimale et maximale prisent par ẽ et le critère d'utilité est donc le suivant.

- Choisir $\tilde{e}$ afin de maximiser:

$$
\int d \bar{F}(e) \gamma(\underline{v} 1\{\tilde{e}<\underline{e}\}-\bar{c}(\tilde{e}), \bar{v} 1\{\tilde{e}<\bar{e}\}-\underline{c}(\tilde{e})) .
$$

La fonction $\gamma$ peut se décomposer de la manière suivante:

$$
\gamma(\underline{a}, \bar{a})=\rho(\underline{a}, \bar{a}) u(\underline{a})+(1-\rho(\underline{a}, \bar{a})) u(\bar{a})
$$

si bien que le modèle de Jaffray réalise une séparation claire entre

- la perception de l'ambiguïté qui est caractérisée par la fonction de croyance,

- l'attitude du régulateur vis-à-vis du risque, caractérisée par la fonction $u$,

- l'attitude du régulateur vis-à-vis de l'ambiguïé, caractérisée par la fonction $\rho \in[0,1]$.

- $\rho(\underline{a}, \bar{a})=1$ correspond à un critère maxmin et donc à l'aversion maximale pour l'ambiguïté,

$-\rho(\underline{a}, \bar{a})=0$ correspond à un critère maxmax, 


$$
\text { - } \rho(\underline{a}, \bar{a})=\rho \in(0,1) \text { correspond au critère de Hurwicz. }
$$

Cette nette séparation entre la perception de l'ambiguïté (la fonction de croyance), l'attitude (du régulateur) vis-à-vis du risque, d'une part et de l'ambiguité, d'autre part, est unique en théorie de la décision: elle permet l'articulation du critère de décision avec l'information objective obtenue sous la forme d'une fonction de croyance.

\subsection{Résumé du critère de décision.}

- Les évènements $A(e)$ de probabilité $\bar{F}(e)$ caractérisent la fonction de croyance, donc l'information objective à la disposition du régulateur,

- le choix d'émissions maximales ẽ est caractérisé par sa valeur maximale $\bar{v} 1\{\tilde{e}<\bar{e}\}-\underline{c}(\tilde{e})$ et sa valeur minimale $\underline{v} 1\{\tilde{e}<\underline{e}\}-\bar{c}(\tilde{e})$ sur $A(e)$,

- l'attitude du régulateur vis-à-vis du risque est caractérisée par la fonction $u$, tandis que l'attitude vis-à-vis de l'ambiguïté est caractérisée par la fonction $\rho:(\underline{a}, \bar{a}) \mapsto \rho(\underline{a}, \bar{a}) \in[0,1]$.

Enfin, le critère de décision est la maximisation de l'expression (2.1). Par exemple, un régulateur neutre au risque et averse à l'ambiguïté choisira $\tilde{e}$ afin de maximiser $\int d \bar{F}(e)(\underline{v} 1\{\tilde{e}<\underline{e}\}-\bar{c}(\tilde{e}))$. Nous avons ainsi un critère simple de décision correspondant à l'étape 4. du programme décrit par Jacques Drèze [4] et qui met en jeu une représentation de l'ambiguïté obtenue à l'aide d'une procédure d'inférence économétrique qui vient remplacer l'étape 3. de ce même programme, l'adaptant ainsi à une situation ambigüe sans en modifier aucunement la philosophie générale.

\section{CONCLUSion}

L'identification partielle dans les modèles d'élicitation de connaissances relatives à un problème de politique environnementale produit une fomulation de l'incertitude sous forme d'une fonction de croyance objective. Le critère de décision de Jaffray est ainsi particulièrement bien adapté à l'articulation entre inférence à l'aide de modèles partiellement identifiés, soit du fait d'observations censurées ou de la spécification incomplète des modèles, et la décision à l'aide de la connaissance qui en est issue. Cette articulation est illustrée de manière simple à l'aide d'un problème de choix du niveau optimal d'émissions toxiques dans un lac. L'exemple stylisée est destiné à servir de support didactique pour mettre en évidence la relation fondamentale entre identification partielle et fonctions de croyance en théorie de la décision. 


\section{REFERENCES}

[1] Stephen Carpenter, David Ludwig, and William Brock. Management of eutrophication for lakes subject to potentially irreversible change. Ecological applications, 9:751-771, 1999.

[2] Gustave Choquet. Theory of capacities. Annales de l'Institut Fourier, 5:131-295, 1954.

[3] Arthur Dempster. Upper and lower probabilities induced by a multi-valued mapping. Annals of Mathematical Statistics, 38:325-339, 1967.

[4] Jacques Drèze. Econometrics and decision theory. Econometrica, 40:1-18, 1972.

[5] Maurice Fréchet. Sur les tableaux de corrélation dont les marges sont données. Annales de l'université de Lyon, Sciences, Section A, 14:53-77, 1951.

[6] Alfred Galichon and Marc Henry. Set identification in models with multiple equilibria. Review of Economic Studies, 78:1264-1298, 2011.

[7] Christian Gouriéroux, Jean-Jacques Laffont, and Alain Monfort. Coherency conditions in simultaneous linear equations models with endogenous switching regimes. Econometrica, 48:675-695, 1980.

[8] Roger Guesnerie. Calcul économique et développement durable. Revue économique, 55:363-382, 2004.

[9] Claude Henry. Orientation du progrès technique et développement durable. Annales d'économie et de statistique, 57:83-108, 2000.

[10] Claude Henry and Marc Henry. Science incertaine et principe de précaution. Revue économique, 54:1277-1290, 2003.

[11] Jean-Charles Hourcade. Estimating the costs of mitigating greenhouse gases. Climate Change: contribution to the second assessment report of the IPCC, 8:263-293, 1995.

[12] Jean-Yves Jaffray. Linear utility theory for belief functions. Operations Research Letters, 8:107$112,1989$.

[13] Edmond Malinvaud. Capital accumulation and efficient allocation of resources. Econometrica, 21:233-268, 1953.

[14] Charles Manski. Identification problems in the social sciences. Harvard University Press, 1995.

[15] Georges Matheron. Random Sets and Integral Geometry. New York: Wiley, 1975.

[16] Leonard Savage. Foundations of Statistics. New York: Wiley, 1954.

[17] Richard Schmalensee. The costs of environmental protection. MIT-CEEPR WP93-015, 1993.

[18] Cédric Villani. Topics in Optimal Transportation. Providence: American Mathematical Society, 2003.

[19] Martin Weitzman. Gamma discounting. American Economic Review, 91:260-271, 2001. 